\title{
The Diameter of Unit Graphs of Rings
}

\author{
Huadong $\mathrm{Su}^{*}$ and Yangjiang Wei
}

\begin{abstract}
Let $R$ be a ring. The unit graph of $R$, denoted by $G(R)$, is the simple graph defined on all elements of $R$, and where two distinct vertices $x$ and $y$ are linked by an edge if and only if $x+y$ is a unit of $R$. The diameter of a simple graph $G$, denoted by $\operatorname{diam}(G)$, is the longest distance between all pairs of vertices of the graph $G$. In the present paper, we prove that for each integer $n \geq 1$, there exists a ring $R$ such that $n \leq \operatorname{diam}(G(R)) \leq 2 n$. We also show that $\operatorname{diam}(G(R)) \in\{1,2,3, \infty\}$ for a ring $R$ with $R / J(R)$ self-injective and classify all those rings with $\operatorname{diam}(G(R))=1,2,3$ and $\infty$, respectively. This extends 12 , Theorem 2 and Corollary 1$]$.
\end{abstract}

\section{Introduction}

The investigation of the interplay between ring theory and graph theory by associating a graph to a ring has attracted considerable attention in past two decades. In 1988, the zero-divisor graph of a commutative ring was first introduced and studied by Beck in [8]. Since then, many authors have studied various forms of zero-divisor graphs associated to rings and other algebraic structures. Generally speaking, studying the zero divisor graph is a way to investigate the ring through the properties of its zero divisors. This is applicable especially when the zero divisors of the ring can be easily identified. This may explain why most of the publications on the zero divisor graph just concern the finite rings. The units of a ring are key elements in determining the structure of the ring, and many properties of a ring are closely connected to these of its units. So it is natural to associate a ring with a graph whose edge relationships rely on units of the ring instead of zero divisors. The unit graph of a ring is such a graph, which is the topics in this paper.

This paper concerns with diameters of unit graphs of rings. The study of diameters of graphs associated with rings is the active topic in this area. For example, Anderson and Livingston [4], Anderson and Mulay [5] investigated the diameter of the zero-divisor graph of a commutative ring, respectively. It was proved that the zero-divisor graph of a commutative ring is always connected with diameter at most three. A similar version for the zero-divisor graph of a commutative semigroup was shown in $[9$ by DeMeyer,

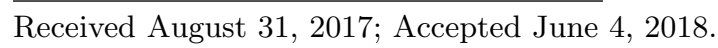

Communicated by Sen-Peng Eu.

2010 Mathematics Subject Classification. Primary: 05C25, 16U60; Secondary: 05C12.

Key words and phrases. unit graph, diameter, $k$-good, unit sum number, self-injective ring.

*Corresponding author. 
McKenzie and Schneider. However, Anderson and Badawi [3] proved that for each integer $n \geq 1$, there exists a ring $R$ such that its total graph has diameter $n$. For the unit graph, Heydari and Nikmehr [12] proved that its diameter is 1,2,3 or $\infty$ for an Artinian ring.

Let $R$ be a ring with identity. Recall that the unit graph of a $\operatorname{ring} R$, denoted $G(R)$, is the simple graph defined on the elements of $R$ with an edge between two distinct vertices $x$ and $y$ if and only if $x+y$ is a unit of $R$. In 1990, the unit graph was first investigated by Grimaldi for $\mathbb{Z}_{n}$, the ring of integers modulo $n$, in [10] where the author considered the degree of a vertex, the Hamilton cycles, the covering number, the independence number and the chromatic polynomial of the graph $G\left(\mathbb{Z}_{n}\right)$. In 2010, Ashrafi, et al. [6] generalized the unit graph $G\left(\mathbb{Z}_{n}\right)$ to $G(R)$ for an arbitrary ring $R$ and obtained various characterization results for finite (commutative) rings regarding connectedness, chromatic index, diameter, girth, and planarity of $G(R)$. Maimani et al. gave the necessary and sufficient conditions for unit graphs to be Hamiltonian in [15]. Heydari and Nikmehr investigated the unit graph of a left Artinian ring in [12]. Afkhami and Khosh-Ahang studied the unit graphs of rings of polynomials and power series in [1]. In 2014, Su and Zhou [18 proved that the girth of $G(R)$ is $3,4,6$ or $\infty$ for an arbitrary ring $R$. Other papers are also devoted to this topic (see, [2, 16, 17]).

In this paper, we study the diameter of the unit graphs, and relate it to the structure of rings. We first determine when $\operatorname{diam}(G(R / J(R)))$ equals $\operatorname{diam}(G(R))$ (see Corollary 3.3). In [6], the authors have shown that $\operatorname{diam}(G(R)) \in\{1,2,3, \infty\}$ for a finite $\operatorname{ring} R$ and later in [12] for a left Artinian ring $R$. We extend this result to rings $R$ with $R / J(R)$ self-injective ring (see Theorem 3.6) and classify the rings $R$ with $R / J(R)$ self-injective with $\operatorname{diam}(G(R))=1,2,3$ or $\infty$, respectively (see Theorem 3.7). We also show that there exists a ring $R$ such that $3<\operatorname{diam}(G(R))<\infty$ (see Corollary 2.7).

Let us first recall some needed notions in graph theory. Let $G$ be a simple graph. A walk is a sequence of vertices and edges, where each edge's endpoints are the preceding and following vertices in the sequence. The length of a walk is the number of edges that it uses. A path in a graph is a walk that has all distinct vertices (except the endpoints). We use $x-y$ to denote two vertices $x$ and $y$ in a graph $G$ are adjacent. A graph $G$ is connected if there is a path between each pair of the vertices of $G$; otherwise, $G$ is disconnected. The distance between two vertices $x$ and $y$, denoted $d(x, y)$, is the length of the shortest path in $G$ beginning at $x$ and ending at $y$. The largest distance between all pairs of vertices of $G$ is called the diameter of $G$, and is denoted by $\operatorname{diam}(G)$. A complete graph is a graph where each vertex is adjacent to all other vertices. Obviously, $G$ is a complete graph if and only if $\operatorname{diam}(G)=1$. We use $K_{m, n}$ and $K_{n}$ to denote the complete bipartite graph with partitions of size $m$ and $n$, and the complete graph of $n$ vertices, respectively.

Throughout the paper, rings are associate and have nonzero identity. Let $R$ be a 
ring. The characteristic of $R$ is denoted by $\operatorname{char}(R)$. We use $J(R)$ to denote the Jacobson radical of $R$ and write $\bar{R}=R / J(R)$ and $\bar{a}=a+J(R) \in \bar{R}$ for $a \in R$. We use $\mathbb{Z}_{n}, R[t]$ and $R[[t]]$ to denote the ring of integers modulo $n$, the polynomial ring over a ring $R$ in the indeterminate $t$, and the formal power series ring over a ring $R$ in the indeterminate $t$, respectively. Recall that a ring $R$ is called right self-injective if, for any (principal) right ideal $I$ of $R$, every homomorphism from $I_{R}$ to $R_{R}$ extends to a homomorphism from $R_{R}$ to $R_{R}$.

\section{Unit graphs with diameter $n$}

As shown in [6], the connectedness of $G(R)$ is relative to whether the ring $R$ is generated additively by its units. So we first recall the following definitions. Let $R$ be a ring and $k$ be a positive integer. An element $r \in R$ is said to be $k$-good if $r=u_{1}+\cdots+u_{k}$ with $u_{i} \in U(R)$ for each $1 \leq i \leq k$. A ring is said to be $k$-good if every element of $R$ is $k$-good. The unit sum number of a ring $R$, denoted by $\mathbf{u}(R)$, is defined to be

(1) $\min \{k \in \mathbb{N} \mid R$ is a $k$-good $\}$, if $R$ is $k$-good for some $k \geq 1$;

(2) $\omega$, if $R$ is not $k$-good for every $k \geq 1$, but each element of $R$ is $k$-good for some $k$;

(3) $\infty$, some element of $R$ is not $k$-good for any $k \geq 1$.

For example, $\mathbf{u}\left(\mathbb{Z}_{3}\right)=2, \mathbf{u}(\mathbb{Z})=\omega$ and $\mathbf{u}(\mathbb{Z}[t])=\infty$. It is clear that if $2 \in U(R)$, then $r \in R$ being $k$-good implies that $r$ is $l$-good for all $l \geq k$. The investigation of rings generated additively by their units started in 1953-1954 when Wolfson [20] and Zelinsky 21 proved independently that every linear transformation of a vector space $V$ over a division ring $D$ is the sum of two nonsingular linear transformations, except when $\operatorname{dim} V=1$ and $D=\mathbb{Z}_{2}$. For the unit sum number of rings, we refer the reader to $13,14,19$.

In [6, Theorem 3.4], the authors characterized when the unit graph of a ring is a complete graph.

Lemma 2.1. 6, Theorem 3.4] Let $R$ be a ring. Then the unit graph $G(R)$ is a complete graph $(\operatorname{diam}(G(R))=1)$ if and only if $R$ is a division ring with $\operatorname{char}(R)=2$.

Lemma 2.2. Let $R$ be a ring and $r \in R$. Then the following hold:

(1) If $r$ is $k$-good, then $d(r, 0) \leq k$;

(2) If $r \neq 0$ and $d(r, 0)=k$, then $r$ is $k$-good but not $l$-good for all $l<k$.

Proof. (1) Let $r=u_{1}+\cdots+u_{k}$, where each $u_{i} \in U(R)$. If $k$ is odd, then $0-u_{1}-\left(-u_{1}-u_{2}\right)-\cdots-\left(-u_{1}-\cdots-u_{k-1}\right)-\left(u_{1}+\cdots+u_{k}\right)=r$ 
is a walk of length $k$. If $k$ is even, then

$$
0-\left(-u_{1}\right)-\left(u_{1}+u_{2}\right)-\cdots-\left(-u_{1}-\cdots-u_{k-1}\right)-\left(u_{1}+\cdots+u_{k}\right)=r
$$

is a walk of length $k$. Therefore, $d(r, 0) \leq k$.

(2) Let $r=x_{0}-x_{1}-x_{2}-\cdots-x_{k}=0$ be a path from $r$ to 0 . Then $u_{i}:=x_{i-1}+x_{i} \in$ $U(R)$ for $1 \leq i \leq k$, so $r=\sum_{i=1}^{k}(-1)^{i+1} u_{i}$. Thus, $r$ is $k$-good. By part (1), we know that $r$ is not $l$-good for all $l<k$.

Proposition 2.3. Let $R$ be a ring that is not a division ring. If $\mathbf{u}(R)=k$, then $\operatorname{diam}(G(R))=k$.

Proof. Let $x, y \in R$. If $k$ is odd, we set $x+y=u_{1}+u_{2}+\cdots+u_{k}$, where each $u_{i}$ is a unit in $R$. Then there exists a walk

$$
x-\left(-x+u_{1}\right)-\left(x-u_{1}-u_{2}\right)-\cdots-\left(x-u_{1}-\cdots-u_{k-1}\right)-\left(-x+u_{1}+\cdots+u_{k}\right)=y
$$

between $x$ and $y$, so $d(x, y) \leq k$. If $k$ is even, we set $y-x=u_{1}+u_{2}+\cdots+u_{k}$, where each $u_{i}$ is a unit in $R$. Then there exists a walk

$$
x-\left(-x-u_{1}\right)-\left(x+u_{1}+u_{2}\right)-\cdots-\left(x-u_{1}-\cdots-u_{k-1}\right)-\left(x+u_{1}+\cdots+u_{k}\right)=y
$$

between $x$ and $y$, so $d(x, y) \leq k$. Thus, $\operatorname{diam}(G(R)) \leq k$.

On the other hand, as $\mathbf{u}(R)=k$, there exists an element $0 \neq r \in R$, such that $r$ is $k$-good but not $l$-good for any $l<k$. Then $d(r, 0)=k$ by Lemma 2.2. Thus, $\operatorname{diam}(G(R))=k$.

The condition that $R$ is not a division ring is necessary. For example, $\mathbf{u}\left(\mathbb{Z}_{2}\right)=\omega$, but $\operatorname{diam}\left(G\left(\mathbb{Z}_{2}\right)\right)=1$. Moreover, the converse of Proposition 2.3 is not true in general. For example, $\operatorname{diam}\left(G\left(\mathbb{Z}_{4}\right)\right)=2$, but $\mathbf{u}\left(\mathbb{Z}_{4}\right)=\omega$.

Proposition 2.4. Let $R$ be a ring with $\operatorname{diam}(G(R))=k \geq 2$. If $2 \in U(R)$, then $\mathbf{u}(R)=k$.

Proof. By Lemma 2.1, $R$ is not a division ring with $\operatorname{char}(R)=2$. If $R$ is a division ring with $\operatorname{char}(R) \neq 2$, then $\operatorname{diam}(G(R))=2$. Note that, in this case, $\mathbf{u}(R)=2$. So the result follows.

Now we assume $R$ is not a division ring. Let $0 \neq r \in R$. If $d(r, 0)=l \leq k$, then by Lemma 2.2(2), we know that $r$ is $l$-good. Since 2 is a unit of $R, r$ is $k$-good and hence $R$ is $k$-good. By Proposition 2.3, $R$ is not $l$-good for all $l<k$, so $\mathbf{u}(R)=k$.

Proposition 2.5. Let $R$ be a ring with $2 \in U(R)$ and $k \geq 2$ an integer. Then $\operatorname{diam}(G(R))$ $=k$ if and only if $\mathbf{u}(R)=k$.

Proof. This follows from Propositions 2.3 and 2.4 . 
We note that, however, in the previous example, every element in $\mathbb{Z}_{4}$ can be expressed as a sum of at most two units. So we recall another slightly different definition which was introduced in [11]. Let $\operatorname{usn}(R)$ be the smallest number $n$ such that every element can be written as the sum of at most $n$ units. If some element of $R$ is not $k$-good for any $k \geq 1$, then $\operatorname{usn}(R)$ is defined to be $\infty$. Note that $\operatorname{usn}(R)$ and $\mathbf{u}(R)$ are different. For example, $\mathbf{u}\left(\mathbb{Z}_{2}\right)=\omega$ and $\operatorname{usn}\left(\mathbb{Z}_{2}\right)=2$.

In [12, Heydari and Nikmehr proved that $\operatorname{diam}(G(R)) \in\{1,2,3, \infty\}$ for an Artinian ring $R$. It is interesting to know whether there exists a $\operatorname{ring} R$ such that $3<\operatorname{diam}(G(R))<$ $\infty$. In [11, Corollary 4], the authors proved that there exists a ring $R$ such that $\operatorname{usn}(R)=n$ for each given $n \geq 2$. This result can be used to show that there exists a ring $R$ such that $3<\operatorname{diam}(G(R))<\infty$.

Theorem 2.6. Let $R$ be a ring but not a division ring. If $\operatorname{usn}(R)=n$, then $n \leq$ $\operatorname{diam}(G(R)) \leq 2 n$.

Proof. We can assume that $\operatorname{usn}(R)=n \geq 2$. Then there exists an element $0 \neq r \in R$, such that $r$ is a sum of $n$ units but not a sum of $m$ units for any $m<n$. We claim that $d(r, 0) \geq n$. If $d(r, 0)=k<n$, then, by Lemma $2.2(2), r$ is $k$-good, a contradiction. So $d(r, 0) \geq n$ and hence $\operatorname{diam}(G(R)) \geq n$. On the other hand, For $x, y \in R$, suppose that $x$ is $k$-good and $y$ is $l$-good. By Lemma $2.2(1), d(x, 0) \leq k \leq n$ and $d(y, 0) \leq l \leq n$, so $d(x, y) \leq k+l \leq 2 n$. This implies that $\operatorname{diam}(G(R)) \leq 2 n$.

Corollary 2.7. There exists a ring $R$ such that $3<\operatorname{diam}(G(R))<\infty$.

Proof. This follows from Theorem 2.6 and [11, Corollary 4].

The condition that $R$ is not a division ring is necessary in Theorem 2.6. For example, $\operatorname{usn}\left(\mathbb{F}_{4}\right)=2$, but $\operatorname{diam}\left(G\left(\mathbb{F}_{4}\right)\right)=1$.

We characterize the rings $R$ with $\operatorname{diam}(G(R))=2$ for ending this section.

Proposition 2.8. Let $R$ be a ring. Then $\operatorname{diam}(G(R))=2$ if and only if $\operatorname{usn}(R)=2$ and $R$ is not a division ring with $\operatorname{char}(R)=2$.

Proof. Assume $\operatorname{diam}(G(R))=2$. Then $R$ is not a division ring with $\operatorname{char}(R)=2$ by Lemma 2.1. For any nonzero nonunit $r$ in $R$, as $\operatorname{diam}(G(R))=2$, we have $d(r, 0)=2$. So $r$ is 2 -good by Lemma $2.2(2)$. So $\operatorname{usn}(R)=2$. Conversely, it is clear that $\operatorname{diam}(G(R)) \geq 2$. For any $x, y \in R$, if $x+y \in U(R)$, then $d(x, y)=1$; if $x+y \notin U(R)$, then $x+y$ is 2-good. So $d(x-y, 0)=2$ and hence $d(x, y)=2$ by Lemma $2.2(1)$. Thus $\operatorname{diam}(G(R))=2$. 


\section{Self-injective rings}

Here, our concern is self-injective rings. In [6], Ashrafi et al. proved that $\operatorname{diam}(G(R))$ is $1,2,3$ or $\infty$ for a finite (commutative) ring $R$, In [12], Heydari and Nikmehr generalized the result to an Artinian ring $R$ and classified all Artinian rings according to diameters of their unit graphs. Our purpose in this section is to generalize these results to rings $R$ with $R / J(R)$ self-injective.

In [12, Remark 1], the authors have pointed out that $\operatorname{diam}(G(\bar{R})) \leq \operatorname{diam}(G(R))$ for any ring $R$. Here, we first determine when the inequality is strict.

Lemma 3.1. Let $R$ be a ring. If $\operatorname{diam}(G(R)) \geq 3$, then $\operatorname{diam}(G(\bar{R}))=\operatorname{diam}(G(R))$.

Proof. Suppose that $\operatorname{diam}(G(R))=\infty$. We need to show that $\operatorname{diam}(G(\bar{R}))=\infty$. Assume to the contrary that $\operatorname{diam}(G(\bar{R}))=m<\infty$. For any $x, y \in R$, if $\bar{x}=\bar{y}$, then $x-y \in J(R)$ and hence $1+x-y \in U(R)$. So we get a path $x-(1-y)-y$ from $x$ to $y$, and so $d(x, y) \leq 2$. If $\bar{x} \neq \bar{y}$, then a path from $\bar{x}$ to $\bar{y}$ deduces a path from $x$ to $y$, which implies that $d(x, y) \leq d(\bar{x}, \bar{y}) \leq m$. So $\operatorname{diam}(G(R))<\infty$, a contradiction.

Suppose that $\operatorname{diam}(G(R)$ is finite and $\operatorname{diam}(G(R))=k \geq 3$. By [12, Remark 1], we only need to show that $\operatorname{diam}(G(\bar{R})) \geq k$. There exist $x, y \in R$, such that $d(x, y)=k$. First we claim that $\bar{x} \neq \bar{y}$. Otherwise, $\bar{x}=\bar{y}$, then $x-y \in J(R)$ and hence $1+x-y \in U(R)$. So $x-(1-y)-y$ is a walk from $x$ to $y$, so $d(x, y) \leq 2$, a contradiction. Assume that $d(\bar{x}, \bar{y})=l<k$ and $\bar{x}-\overline{x_{1}}-\overline{x_{2}}-\cdots-\overline{x_{l-1}}-\bar{y}$ is a path from $\bar{x}$ to $\bar{y}$. Then $x-x_{1}-x_{2}-$ $\cdots-x_{l-1}-y$ is path of length $l$, so $d(x, y) \leq l<k$, a contradiction. Thus, $d(\bar{x}, \bar{y}) \geq k$. This implies $\operatorname{diam}(G(\bar{R})) \geq k$.

Therefore, $\operatorname{diam}(G(\bar{R}))=\operatorname{diam}(G(R))$.

Now, we determine when $\operatorname{diam}(G(\bar{R}))<\operatorname{diam}(G(R))$.

Theorem 3.2. Let $R$ be a ring. Then the following are equivalent:

(1) $\operatorname{diam}(G(\bar{R}))<\operatorname{diam}(G(R))$.

(2) $R$ is a local ring with $J(R) \neq 0$ and $2 \in J(R)$.

(3) $\operatorname{diam}(G(R))=2$ and $\operatorname{diam}(G(\bar{R}))=1$.

Proof. $(1) \Rightarrow(2)$. Suppose that $\operatorname{diam}(G(\bar{R}))<\operatorname{diam}(G(R))$. By Lemma 3.1, $\operatorname{diam}(G(R))$ $\leq 2$. Note that $\operatorname{diam}(G(R))=1$ implies $\operatorname{diam}(G(\bar{R}))=1$. Thus, we have $\operatorname{diam}(G(R))=2$ and $\operatorname{diam}(G(\bar{R}))=1$. So $J(R) \neq 0$, and by Lemma 2.1, $\bar{R}$ is a division ring with $\operatorname{char}(\bar{R})=2$. Therefore, $R$ is a local ring with $J(R) \neq 0$ and $2 \in J(R)$.

$(2) \Rightarrow(3)$. Suppose that $R$ is a local ring with $J(R) \neq 0$ and $2 \in J(R)$. Then $R / J(R)$ is a division ring and $\operatorname{char}(\bar{R})=2$. By Lemma 2.1, it follows that $G(\bar{R})$ is a complete 
graph and hence $\operatorname{diam}(G(\bar{R}))=1$. On the other hand, for any $r \in R$, either $r \in J(R)$ or $r \in U(R)$. For any two distinct elements $a, b \in R$, if $a+b \in U(R)$, then $d(a, b)=1$. Suppose that $a+b \in J(R)$. If $a \in J(R)$, then $b \in J(R)$, and we have a path $a-1-b$, so $d(a, b)=2$ (note that since $J(R) \neq 0$, such $a, b$ do exist); if $a \in U(R)$, then $b \in U(R)$, and we have a path $a-(a+b)-b$, so $d(a, b)=2$. Hence $\operatorname{diam}(G(R))=2$.

$(3) \Rightarrow(1)$. It is clear.

Corollary 3.3. Let $R$ be a ring. Then $\operatorname{diam}(G(\bar{R}))=\operatorname{diam}(G(R))$ if and only if one of the following holds:

(1) $R$ is not a local ring.

(2) $R$ is a local ring with $2 \in U(R)$.

(3) $R$ is a division ring.

In [13, Theorem 6], Khurana and Srivastava determined the unit sum number $\mathbf{u}(R)$ of a regular right self-injective ring $R$. We use the notion usn $(R)$ to restate the theorem below.

Lemma 3.4. [13 Let $R$ be a regular self-injective ring. Then $\operatorname{usn}(R)=2,3$ or $\infty$. Moreover,

(1) $\operatorname{usn}(R)=2$ if and only if $R$ has no nonzero Boolean ring as a ring direct summand or $R \cong \mathbb{Z}_{2}$.

(2) $\operatorname{usn}(R)=3$ if and only if $R \varsubsetneqq \mathbb{Z}_{2}$ and $R$ has $\mathbb{Z}_{2}$, but no Boolean ring with more than two elements, as a ring direct summand.

(3) $\operatorname{usn}(R)=\infty$ if and only if $R$ has a Boolean ring with more than two elements as a ring direct summand.

Lemma 3.5. Let $R$ be a regular right self-injective ring. Then $\operatorname{diam}(G(R)) \in\{1,2,3, \infty\}$.

Proof. By [13, Theorem 6], $\mathbf{u}(R)=2, \omega$ or $\infty$. Suppose that $\mathbf{u}(R)=2$. If $R$ is not a division ring, then $\operatorname{diam}(G(R)=2$ by Proposition 2.3. If $R$ is a division ring, it is clear that $\operatorname{diam}(G(R)) \leq 2$.

Suppose that $\mathbf{u}(R)=\omega$. Then, by $[13$, Theorem $6(2)]$, we may assume that $R=$ $R_{1} \times \mathbb{Z}_{2}$, where $\mathbf{u}\left(R_{1}\right)=1$ or 2 . If $\mathbf{u}\left(R_{1}\right)=1, R_{1}$ is a trivial ring and $R=\mathbb{Z}_{2}$ and so $\operatorname{diam}(G(R))=1$. Now suppose that $\mathbf{u}\left(R_{1}\right)=2$ and let $x, y \in G(R)$. If $x=\left(x_{1}, 0\right)$ and $y=\left(y_{1}, 0\right)$, then there exists $z_{1} \in R_{1}$, such that $x_{1}+z_{1}$ and $z_{1}+y_{1}$ are units in $R_{1}$. So a path $\left(x_{1}, 0\right)-\left(z_{1}, 1\right)-\left(y_{1}, 0\right)$ from $\left(x_{1}, 0\right)$ to $\left(y_{1}, 0\right)$ deduces $d(x, y) \leq 2$; if $x=\left(x_{1}, 1\right)$ and $y=\left(y_{1}, 1\right)$, a similar argument shows that $d(x, y) \leq 2$; if $x=\left(x_{1}, 0\right)$ and $y=\left(y_{1}, 1\right)$, then 
there exists $z_{1} \in R_{1}$, such that $x_{1}+z_{1}$ is a unit in $R_{1}$. With a similar argument, we have a path $\left(x_{1}, 0\right)-\left(z_{1}, 1\right)-\left(w_{1}, 0\right)-\left(y_{1}, 1\right)$ and hence $d(x, y) \leq 3$. So $\operatorname{diam}(G(R)) \leq 3$ always holds.

Suppose that $\mathbf{u}(R)=\infty$. By [6, Theorem 4.3], we know that $G(R)$ is disconnected. So $\operatorname{diam}(G(R)=\infty$. The proof is complete.

Theorem 3.6. Let $R$ be a ring with $R / J(R)$ right self-injective (in particular, $R$ is right self-injective). Then $\operatorname{diam}(G(R)) \in\{1,2,3, \infty\}$.

Proof. We know that in this case $\bar{R}=R / J(R)$ is a regular right self-injective ring. By Lemma 3.5, we have $\operatorname{diam}(\bar{R}) \in\{1,2,3, \infty\}$. By Lemma 3.1, we get $\operatorname{diam}(G(R)) \in$ $\{1,2,3, \infty\}$.

Theorem 3.7. Let $R$ be a ring with $R / J(R)$ right self-injective (in particular, $R$ is right self-injective). Then the following hold:

(1) $\operatorname{diam}(G(R))=1$ if and only if $R$ is a division ring with $\operatorname{char}(R)=2$.

(2) $\operatorname{diam}(G(R))=2$ if and only if $R$ is not a division ring with $\operatorname{char}(R)=2$ and one of following holds:

(i) $\bar{R}$ has no nonzero Boolean ring as a ring direct summand.

(ii) $\bar{R} \cong \mathbb{Z}_{2}$.

(3) $\operatorname{diam}(G(R))=3$ if and only if $\bar{R} ¥ \mathbb{Z}_{2}$ and $\bar{R}$ has $\mathbb{Z}_{2}$, but no Boolean ring with more than two elements, as a ring direct summand.

(4) $\operatorname{diam}(G(R))=\infty$ if and only if $\bar{R}$ has a Boolean ring with more than two elements as a ring direct summand.

Proof. (1) This follows from Lemma 2.1

Next, we assume that $R$ is not a division ring with $\operatorname{char}(R)=2$ and prove (2), (3) and (4) together. Note that $\bar{R}$ is a regular right self-injective ring. So $\mathbf{u}(\bar{R})=2, \omega$ or $\infty$ by [13, Theorem 6]. To complete the proof, we determine the diameter for each case.

Case 1: $\mathbf{u}(\bar{R})=2$. In this case, $\bar{R}$ has no nonzero Boolean ring as a ring direct summand or $\bar{R} \cong \mathbb{Z}_{2}$ by Lemma 3.4 . Note that $\operatorname{diam}(G(\bar{R})) \in\{1,2\}$. So $\operatorname{diam}(G(R))=2$ by Lemma 3.1 .

Case 2: $\mathbf{u}(\bar{R})=\omega$. If $\bar{R} \cong \mathbb{Z}_{2}$, then $G(R)$ is a complete bipartite graph. So $\operatorname{diam}(G(R))=2$. If $\bar{R} ¥ \mathbb{Z}_{2}$, in this case, usn $(\bar{R})=3$, so $\operatorname{diam}(G(\bar{R}))=3$ as we shown in Lemma 3.5. Thus $\operatorname{diam}(G(R))=3$ by Lemma 3.1 .

Case 3: $\mathbf{u}(\bar{R})=\infty$. Then $G(\bar{R})$ is disconnected by [6, Theorem 4.3]. So $\operatorname{diam}(G(\bar{R}))=$ $\infty$. Thus $\operatorname{diam}(G(R))=\infty$ by Lemma 3.1 . 


\section{Acknowledgments}

This work was supported by the National Natural Science Foundation of China (11661013, 11661014, 11461010), the Guangxi Natural Sciences Foundation (2016GXNSFCA380014, 2015GXNSFAA139009, 2016GXSFDA380017), Guangxi Science Research and Technology Development Project (1599005-2-13), and the Scientific Research Foundation of Guangxi Educational Committee (KY2016YB280).

\section{References}

[1] M. Afkhami and F. Khosh-Ahang, Unit graphs of rings of polynomials and power series, Arab. J. Math. 2 (2013), no. 3, 233-246.

[2] S. Akbari, E. Estaji and M. R. Khorsandi, On the unit graph of a non-commutative ring, Algebra Colloq. 22 (2015), Special Issue no. 1, 817-822.

[3] D. F. Anderson and A. Badawi, The total graph of a commutative ring, J. Algebra 320 (2008), no. 7, 2706-2719.

[4] D. F. Anderson and P. S. Livingston, The zero-divisor graph of a commutative ring, J. Algebra 217 (1999), no. 2, 434-447.

[5] D. F. Anderson and S. B. Mulay, On the diameter and girth of a zero-divisor graph, J. Pure Appl. Algebra 210 (2007), no. 2, 543-550.

[6] N. Ashrafi, H. R. Maimani, M. R. Pournaki and S. Yassemi, Unit graphs associated with rings, Comm. Algebra 38 (2010), no. 8, 2851-2871.

[7] N. Ashrafi and P. Vámos, On the unit sum number of some rings, Q. J. Math. 56 (2005), no. 1, 1-12.

[8] I. Beck, Coloring of commutative rings, J. Algebra 116 (1988), no. 1, 208-226.

[9] F. R. DeMeyer, T. McKenzie and K. Schneider, The zero-divisor graph of a commutative semigroup, Semigroup Forum 65 (2002), no. 2, 206-214.

[10] R. P. Grimaldi, Graphs from rings, Proceedings of the Twentieth Southeastern Conference on Combinatorics, Graph Theory, and Computing (Boca Raton, FL, 1989), Congr. Numer. 71 (1990), 95-103.

[11] B. Herwig and M. Ziegler, A remark on sums of units, Arch. Math. (Basel) 79 (2002), no. $6,430-431$. 
[12] F. Heydari and M. J. Nikmehr, The unit graph of a left Artinian ring, Acta Math. Hungar. 139 (2013), no. 1-2, 134-146.

[13] D. Khurana and A. K. Srivastava, Unit sum numbers of right self-injective rings, Bull. Austral. Math. Soc. 75 (2007), no. 3, 355-360.

[14] _ Right self-injective rings in which every element is a sum of two units, J. Algebra Appl. 6 (2007), no. 2, 281-286.

[15] H. R. Maimani, M. R. Pournaki and S. Yassemi, Necessary and sufficient conditions for unit graphs to be Hamiltonian, Pacific J. Math. 249 (2011), no. 2, 419-429.

[16] H. Su, K. Noguchi and Y. Zhou, Finite commutative rings with higher genus unit graphs, J. Algebra Appl. 14 (2015), no. 1, 1550002, 14 pp.

[17] H. Su, G. Tang and Y. Zhou, Rings whose unit graphs are planar, Publ. Math. Debrecen 86 (2015), no. 3-4, 363-376.

[18] H. Su and Y. Zhou, On the girth of the unit graph of a ring, J. Algebra Appl. 13 (2014), no. 2, 1350082, 12 pp.

[19] P. Vámos, 2-good rings, Q. J. Math. 56 (2005), no. 3, 417-430.

[20] K. G. Wolfson, An ideal-theoretic characterization of the ring of all linear transformations, Amer. J. Math. 75 (1953), 358-386.

[21] D. Zelinsky, Every linear transformation is a sum of nonsingular ones, Proc. Amer. Math. Soc. 5 (1954), 627-630.

Huadong Su and Yangjiang Wei

School of Mathematical and Statistics Sciences, Guangxi Teachers Education University, Nanning, Guangxi, 530023, P. R. China

E-mail address: huadongsu@sohu.com, gus02@163.com 